Disponible en ligne : www.cahiersagricultures.fr

\title{
Comprendre la contribution de la recherche à l'innovation collective par l'exploration de mécanismes de renforcement de capacité
}

\author{
Aurélie Toillier ${ }^{1,2, *}$, Agathe Devaux-Spartakis ${ }^{2,3}$, Guy Faure ${ }^{2,3}$, Danielle Barret ${ }^{4}$ et Catherine Marquié ${ }^{5,6}$ \\ ${ }^{1}$ CIRAD, UMR INNOVATION, BP 596, Ouagadougou, Burkina-Faso \\ 2 INNOVATION, Univ Montpellier, 34090 Montpellier, France \\ 3 CIRAD, UMR INNOVATION, 34398 Montpellier, France \\ ${ }^{4}$ CIRAD, DGDRS, 75116 Paris, France \\ ${ }^{5}$ CIRAD, UPR AIDA, 34398 Montpellier, France \\ ${ }^{6}$ AIDA, Univ Montpellier, 34090 Montpellier, France
}

\begin{abstract}
Résumé - Le renforcement des capacités à innover apparaît comme un nouveau moyen pour assurer un développement durable dans les pays du Sud. Dans le secteur de l'agriculture, l'innovation est essentiellement collective, ce qui appelle les chercheurs à sortir de leur rôle de producteurs de connaissances pour s'engager auprès des acteurs en situation d'innovation. Si une diversité de pratiques de recherche engagée sont apparues, il n'existe cependant pas aujourd'hui une vision claire des différentes façons dont les chercheurs contribuent à l'innovation. L'objectif de cet article est d'identifier les différentes modalités de contribution des chercheurs au renforcement des capacités à innover. Pour cela, les auteurs ont développé un cadre d'analyse ex post qui met en perspective deux corpus de littérature, sur l'apprentissage et le management de l'innovation. Ce cadre permet de caractériser des séquences de situations d'apprentissage et une diversité de postures des chercheurs aux différentes étapes de l'innovation pour rendre compte de leurs contributions. À partir d'une étude approfondie de treize cas d'innovation dans lesquels le Centre de coopération internationale en recherche agronomique pour le développement (CIRAD) s'est engagé avec ses partenaires de recherche du Sud, quatre types de contribution des chercheurs au renforcement des capacités à innover ont été identifiés : faciliter des apprentissages de façon non supervisée, planifier et encadrer des apprentissages, créer des besoins d'apprentissage et y répondre pas à pas, se laisser guider par l'exploration et les besoins des utilisateurs. Nos résultats suggèrent qu'une gestion stratégique des processus d'innovation par les organismes de recherche pourrait être rendue possible par le suivi et l'évaluation des situations d'apprentissage, d'une part de façon à renforcer les capacités à innover des chercheurs eux-mêmes et $\mathrm{d}$ 'autre part pour agencer au mieux les compétences et ressources disponibles, faire évoluer les mandats des chercheurs et rationaliser leurs investissements.
\end{abstract}

Mots clés : innovation collective / renforcement de capacité / situation d'apprentissage / recherche agricole / CIRAD

\begin{abstract}
Understanding the contribution of research to collective innovation through the exploration of capacity development mechanisms. Capacity development for innovation is emerging as a new way to ensure sustainable development in developing countries. In the agricultural sector, innovation is essentially collective, which calls on researchers to step out of their role as producers of knowledge in order to engage with innovating actors. While a diversity of engaged research practices has emerged, there is not yet a clear understanding of the different ways in which researchers contribute to innovation. The aim of this paper is to identify the types of contribution of researchers to capacitydevelopment for innovation. To this end, the authors have developed an ex post analytical framework that puts into perspective two corpus of literature: on learning and management of innovation. This framework makes it possible to characterize sequences of learning situations and a variety of postures of researchers at different stages of innovation in order to account for their contributions. Based on an in-depth study of thirteen innovation cases in which the French Agricultural Centre for International Development (CIRAD) was engaged with its Southern research partners, four types of contribution of researchers to capacity
\end{abstract}

$\overline{\text { *Auteur de correspondance }}$ : aurelie.toillier@cirad.fr 
development to innovate have been identified: to facilitate learning in an unsupervised way; to plan and manage learning processes; to create and respond to learning needs step by step; to be guided by the exploration and needs of end-users. Our results suggest that strategic management of innovation processes by research organizations could be made possible by the monitoring and evaluation of learning situations, on the one hand to strengthen researchers' capacity to innovate, and on the other hand, to better coordinate the skills and resources available, to change the mandates of researchers and to rationalize their investments.

Keywords: collective innovation / capacity development / learning situation / agricultural research / Cirad

\section{Introduction}

Le renforcement des capacités à innover $(\mathrm{RCI})$ des acteurs du développement agricole apparaît comme une voie de développement durable, qui est soutenue par plusieurs agences de coopération internationale (Martin, 2013; TAP, 2016; World Bank, 2007), mais aussi par de nombreux gouvernements nationaux avec l'émergence de politiques dédiées à l'innovation.

Le RCI s'inscrit dans une trajectoire d'évolution des paradigmes d'appui au développement agricole, qui ont évolué au fur et à mesure de la prise de conscience de la complexité des situations, de l'importance de savoir s'adapter aux aléas et prendre des décisions en situation d'incertitude (Hall, 2005; Spielman et al., 2009). Ainsi le terme de «renforcement de capacité» employé par toutes les agences internationales d'appui au développement depuis le milieu des années 90 (Lusthaus et al., 1999) a d'abord consisté en des formations pratiques au champ, à de nouvelles technologies conçues par des chercheurs, puis a visé la résolution de problèmes en facilitant des apprentissages sociaux par des méthodes participatives de conception de solutions associant les différents acteurs concernés, et enfin le co-apprentissage entre tous les acteurs permettant in fine de s'adapter à toute situation en créant les nouveautés nécessaires (Klerkx et al., 2012). Si différents déterminants des capacités à innover sont illustrés dans la littérature - comme la capacité à s'engager, à développer une vision collective des enjeux, à collaborer ou à expérimenter(Woodhill, 2010 ; Leeuwis et al., 2014), il s'agit toujours, in fine, de rendre les individus et les organisations plus autonomes dans l'acquisition et l'usage de nouvelles connaissances utiles à l'atteinte de leurs objectifs. La capacité à innover apparaît alors comme un gage ultime de durabilité.

Ces évolutions dans la façon de penser l'appui au développement agricole ont des implications sur la façon dont les organisations de recherche peuvent conduire des travaux qui contribuent à l'innovation et qui aient des impacts positifs sur le développement (Turner et al., 2016). Le modèle de la recherche partenariale aujourd'hui en vigueur dans plusieurs instituts de recherche finalisée pour le développement, comme le Centre de coopération internationale en recherche agronomique pour le développement (CIRAD), l'Institut de recherche pour le développement (IRD) ou l'Institut national de la recherche agronomique (INRA) en France, permet de mettre en relation les chercheurs avec les acteurs du monde économiques et leurs besoins d'innovation, mais précise mal les modalités de collaboration entre les partenaires au cours d'un projet d'innovation, ainsi que les contributions spécifiques des chercheurs (Audoux et Gillet, 2011). Dans ce contexte, le CIRAD, à travers son programme ImpresS (Hainzelin et al., 2017) s'interroge aujourd'hui sur la manière dont il peut mieux mobiliser ses chercheurs pour augmenter sa contribution à l'innovation et au développement durable.

Pour accompagner l'innovation collective, il ne s'agit plus seulement de fournir des connaissances sur des procédés techniques ou de former des techniciens, mais aussi de fournir des méthodes et connaissances pour aider à organiser la production de connaissances et les échanges entre acteurs (Laperche et al., 2008), faciliter le co-apprentissage (Toillier et al., 2016) ou encore co-construire l'innovation (Vall et al., 2016), de façon à placer les utilisateurs finaux en acteurs de leur propre développement, dépassant ainsi la posture classique du chercheur - intervenant-légitime, neutre, équidistant (Pichault et al., 2008). Par ailleurs, un processus d'innovation est ponctué d'étapes au cours desquelles les besoins en nouvelles connaissances diffèrent (Hall et Andriani, 2003) ainsi que la nature des acteurs à impliquer (Lamers et al., 2017). Quels rôles peuvent ou doivent jouer les chercheurs au cours de ces étapes? Comment évaluer leur(s) contribution(s) à l'ensemble du processus d'innovation? Les nombreuses études décrivant les différentes situations de co-production de connaissances entre chercheurs et acteurs du développement ne proposent pas de vision dynamique de ces situations en lien avec la progression de l'innovation. Elles ne permettent pas non plus de comprendre s'il existe des mécanismes particuliers de renforcement des capacités à innover qui mènent à l'impact.

À la lumière des approches théoriques de l'apprentissage chez les adultes et de travaux sur le management de l'innovation, nous avons construit un cadre d'analyse pour explorer la diversité et la succession de situations d'apprentissage dans un processus d'innovation collectif ainsi que les postures des chercheurs dans chacune de ces situations. Il a été appliqué à treize cas d'étude au Sud qui ont impliqué le CIRAD et ses partenaires de recherche dans chacun des pays concernés. Cette analyse s'inscrit dans le cadre du programme ImpresS d'évaluation de l'impact de la recherche mené par le CIRAD. Nous présentons dans un premier temps notre démarche d'analyse fondée sur une caractérisation participative a posteriori des situations d'apprentissage et leur place sur la trajectoire de l'innovation. Après avoir identifié la diversité des types de contribution des chercheurs aux mécanismes de RCI, nous interrogeons l'utilité de nos résultats pour aider les organisations de recherche à augmenter leur impact.

\section{Cadre d'analyse}

\subsection{Le $\mathrm{RCl}$ vu comme une succession de situations d'apprentissage}

Capacité à innover et apprentissage sont indissociables. Par définition, l'innovation est une réponse inédite à un ensemble de problèmes ou de besoins qui nécessite, entre autres, de la créativité, de l'expérimentation, de l'adaptation, de l'évaluation 

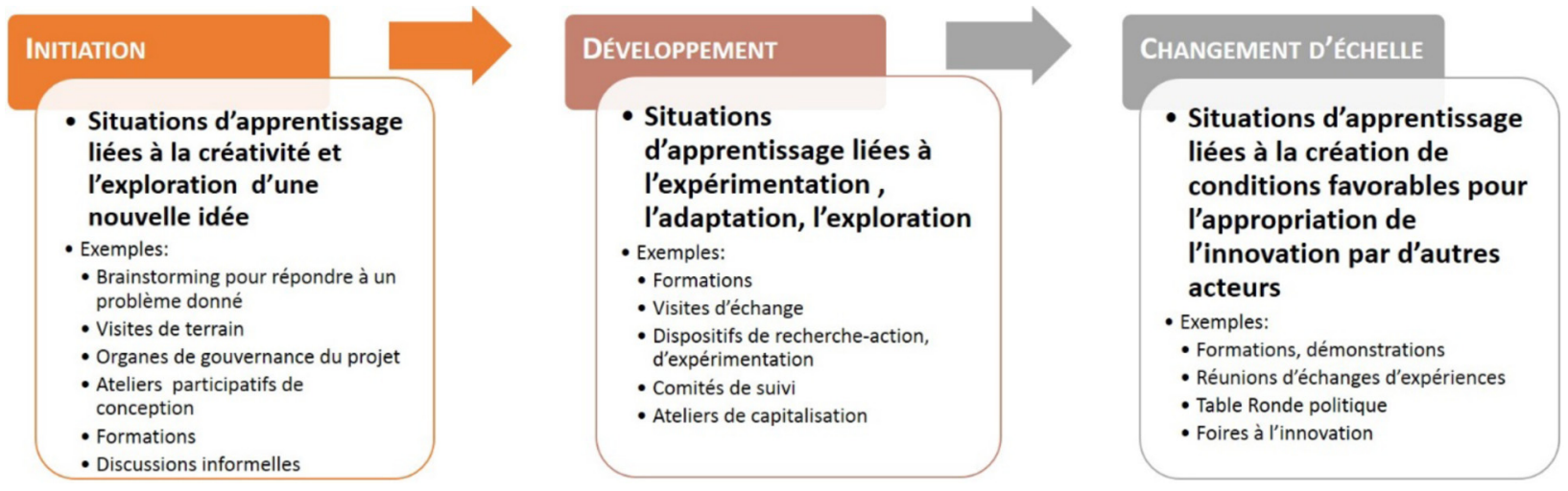

Fig. 1. Exemples de situations d'apprentissage sur une trajectoire d'innovation.

Fig. 1. Examples of learning situations on an innovation trajectory.



Fig. 2. La situation d'apprentissage.

Fig. 2. The learning situation.

mais aussi de savoir mobiliser un ensemble spécifique de moyens humains, matériels et financiers à chacune de ces étapes. La littérature sur le management de l'innovation fait état de corrélations entre ces étapes et la nature des apprentissages requis (Beckman et Michael, 2007). En phase d'initiation, il s'agit principalement d'apprentissages visant à organiser et faire collaborer un collectif pour susciter sa créativité puis l'engager à explorer collectivement une idée nouvelle (Fig. 1). Lors du développement de l'innovation, on parle d'apprentissages d'exploitation en valorisant des connaissances déjà validées ou d'exploration lorsqu'il s'agit de résoudre les problèmes rencontrés en créant de nouvelles connaissances. Ils conduisent les apprenants à progresser dans la conception de l'innovationproduit, ainsi qu'à apprendre à apprendre. Enfin, lors du changement d'échelle, ce sont des apprentissages institutionnels ou organisationnels qui permettent de créer les conditions nécessaires à l'usage et à l'appropriation de l'innovation par d'autres acteurs que ceux qui sont à l'origine de l'innovation.

Nous nous situons dans une perspective où l'on considère que l'apprentissage est intrinsèquement lié à la situation de travail dans laquelle il se produit. Engestrom (1999) montre que lorsqu'un collectif interagit dans un cadre organisé pour résoudre des problèmes ou mettre en œuvre des activités, l'apprentissage et l'innovation naissent des tensions, de confrontations mais aussi de collaborations, liées aux interactions complexes entre les différents acteurs impliqués, l'objet de leur activité commune et la finalité visée.

On propose de définir une situation d'apprentissage collectif (SA) à partir de quatre éléments (Fig. 2): les apprenants, les objets d'apprentissage, le collectif avec lequel les apprenants interagissent au sein du projet d'innovation et qui partagent les mêmes objectifs, et la finalité de la SA qui rend compte des intentions des acteurs. La finalité est révélée par les produits de la situation d'apprentissage (documents, prototype, décisions prises, nouvelles capacités acquises) et par le sens donné par les acteurs eux-mêmes à leurs activités. On considère que la conduite d'un processus d'innovation via un ensemble de projets de Recherche-Développement (R\&D) crée une multitude de situations d'apprentissage particulières, qui ne sont pas toujours prévisibles ou planifiées car un projet d'innovation requiert des ajustements progressifs pour résoudre des problèmes souvent inédits.

\subsection{Localiser les SA sur la trajectoire de l'innovation}

La contribution d'une SA au processus d'innovation est identifiée par sa finalité et par sa position sur la trajectoire du processus d'innovation. On considère que la combinaison de SA reconstituée le long de la trajectoire de l'innovation peut constituer un mécanisme de RCI dans la mesure où les capacités produites sont apparues nécessaires aux acteurs concernés à la progression de l'innovation. Afin de mettre en évidence ce mécanisme, nous nous référons aux boucles d'apprentissage expérientiel de Kolb et al. (2001) qui montrent comment l'enchaînement de situations d'apprentissage - liées à des actions ou des moments de réflexion-conceptualisation dans une perspective expérimentale - permet à des adultes de progresser individuellement et collectivement par le partage 


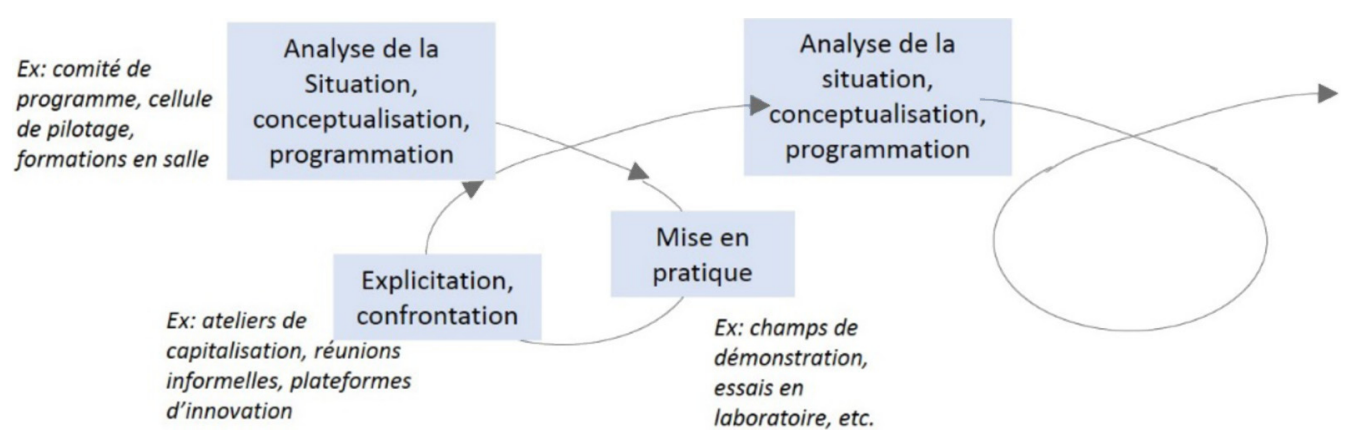

Fig. 3. Boucles de progrès lors d'un processus d'innovation (d'après les boucles d'apprentissage expérientiel de Kolb et al., 2001).

Fig. 3. Progress loops in an innovation process (from experiential learning loops from Kolb et al., 2001).

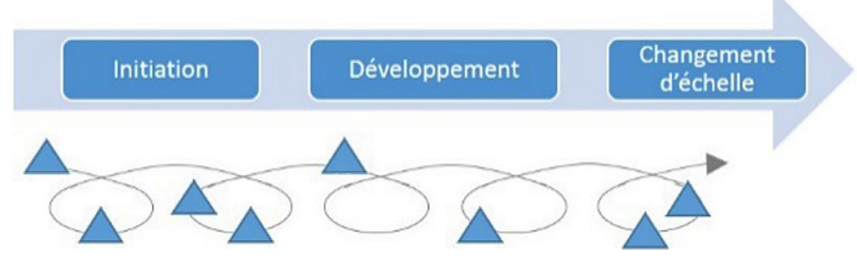

Fig. 4. Trois niveaux d'analyse : trajectoire de l'innovation, situations d'apprentissage et boucles de progrès.

Fig. 4. Three analytical levels: innovation trajectory, learning situation and progress loops.

d'idées, de résultats et d'analyses critiques. On considère que les SA doivent participer à ces boucles de progrès pour contribuer au processus d'innovation (Fig. 3).

La figure 4 synthétise les différents niveaux d'analyse retenus: situations d'apprentissage, boucles de progrès et étapes du processus d'innovation.

\subsection{La posture du chercheur dans les SA comme indicateur de sa contribution}

La qualification de la posture du chercheur dans une SA doit permettre de comprendre sa contribution au RCI. On entend par posture le rôle qu'endosse le chercheur par rapport aux finalités de la SA, sa position vis-à-vis des autres acteurs de la SA et le contenu de ses apports. Une revue de littérature nous a permis d'identifier une diversité de postures dans les recherches partenariales (Tab. 1): intervenant, entrepreneur, explorateur, expert, formateur ou simplement apprenant. Les définitions données nous permettent d'identifier un ensemble d'activités qu'un chercheur est susceptible de mettre en œuvre dans une SA pour contribuer à des apprentissages.

L'évaluation de la contribution des chercheurs aux mécanismes de RCI passe par l'identification de la finalité de la SA dans lequel le chercheur est impliqué et de la nature de ses activités.

\section{Caractériser et comparer treize études de cas}

Treize cas d'innovation impliquant le Cirad et ses partenaires de recherche du Sud ont été sélectionnés (Tab. 2) pour leurs impacts positifs reconnus sur le développement (Triomphe et al., 2015 ; Hainzelin et al., 2017). Ils représentent une diversité de domaines d'innovation (innovations techniques, sociales, organisationnelles, innovations de service).

La méthode d'identification et de caractérisation des trajectoires d'innovation et des situations d'apprentissage a été commune aux treize cas et mise en œuvre par des étudiants via des ateliers participatifs d'auto-évaluation et des entretiens individuels auprès des acteurs les plus impliqués dans le processus d'innovation, indifféremment lors des différentes phases (initiation, développement, changement d'échelle), notamment les chercheurs du Cirad et leurs partenaires. Il leur a été demandé d'identifier les SA qui ont été les plus marquantes, c'est-à-dire celles qui leur ont permis d'acquérir certaines capacités qui ont été selon eux indispensables à la réussite de l'innovation. Elles ont été décrites (type d'activité menée, sa date ou période, sa finalité, les types d'apprenants, la posture des chercheurs, la nature des capacités renforcées, les progrès réalisés grâce aux nouvelles capacités) par des enquêtes ou des focus groupes réunissant une même catégorie d'acteurs (par exemple, des producteurs, des acteurs du service public ou des chercheurs). Le nombre d'enquêtes peut varier d'un cas à l'autre en fonction de la quantité et de la qualité des données sur le processus d'innovation dont disposaient déjà les équipes projets au démarrage de cette étude (Tab. 3). D'après Petticrew et Roberts (2003), l'analyse approfondie de situations concrètes d'action en croisant plusieurs sources d'information, permet de produire des preuves suffisantes de l'existence d'un mécanisme et de sa contribution à des résultats observables. Pour chaque cas d'étude, des analyses croisées des données primaires (ateliers multi-acteurs, entretiens semi-directifs) avec des données secondaires (rapports de projets ou d'ateliers, publications, rapports de stage, bases de données de projets) ont été réalisées afin de valider les trajectoires d'innovation.

Les SA ont été classées en fonction de leurs finalités, du type d'activités menées et de la phase du processus d'innovation, afin d'analyser la fréquence des postures des chercheurs dans des SA similaires (Tab. 4). La posture dominante des chercheurs dans un mécanisme de RCI est déduite de la fréquence de leurs postures dans chaque SA. La combinaison d'une posture dominante, des principaux apprenants et des objets d'apprentissage nous a permis de nommer des types de contributions.

Le classement des SA et l'analyse des types de contribution ont été faits de façon itérative par un groupe de chercheurs non impliqués dans les cas étudiés, et validé ensuite par les chercheurs impliqués, de façon à rendre plus robuste le classement. Les données ont été organisées dans une unique base de données permettant des traitements transversaux. 
Tableau 1. Postures du chercheur dans une situation d'apprentissage au cours d'un processus d'innovation.

Table 1. Postures of the researcher in a learning situation during an innovation process.

\begin{tabular}{|c|c|c|}
\hline $\begin{array}{l}\text { Postures du } \\
\text { chercheur }\end{array}$ & Définition & Références \\
\hline Intervenant & $\begin{array}{l}\text { Posture d'observation, d'analyse et de conceptualisation - légitime, } \\
\text { neutre, équidistant, reformulant la demande d'un système client }\end{array}$ & Pichault et al., 2008; Perez, 2008 \\
\hline Entrepreneur & $\begin{array}{l}\text { Initiateur, concepteur, rédacteur de projet, mobilisateur des partenaires } \\
\text { Facilitateur des interactions au sein d'un groupe de travail, fournisseur } \\
\text { d'expertise méthodologique, organisationnelle, sociale, et juridique, } \\
\text { évaluateur des processus mis en œuvre, et responsable du travail de } \\
\text { théorisation mené tout au long du projet }\end{array}$ & Dimaggio, 1988; Xhauflair et Pichault, 2011 \\
\hline Explorateur & $\begin{array}{l}\text { Organise l'exploration scientifique, définit les objectifs et le protocole, } \\
\text { la démarche et produit des nouvelles connaissances }\end{array}$ & Foray, 1994 ; Delma et al., 2016 \\
\hline Expert & $\begin{array}{l}\text { Fournit des connaissances et une analyse éclairée d'un problème } \\
\text { particulier à la demande d'un client, en portant ses propres valeurs, } \\
\text { idéologie et points de vue }\end{array}$ & Draetta et Labarthe, 2011 \\
\hline
\end{tabular}

Tableau 2. Présentation des cas d'étude.

Table 2. Presentation of case studies.

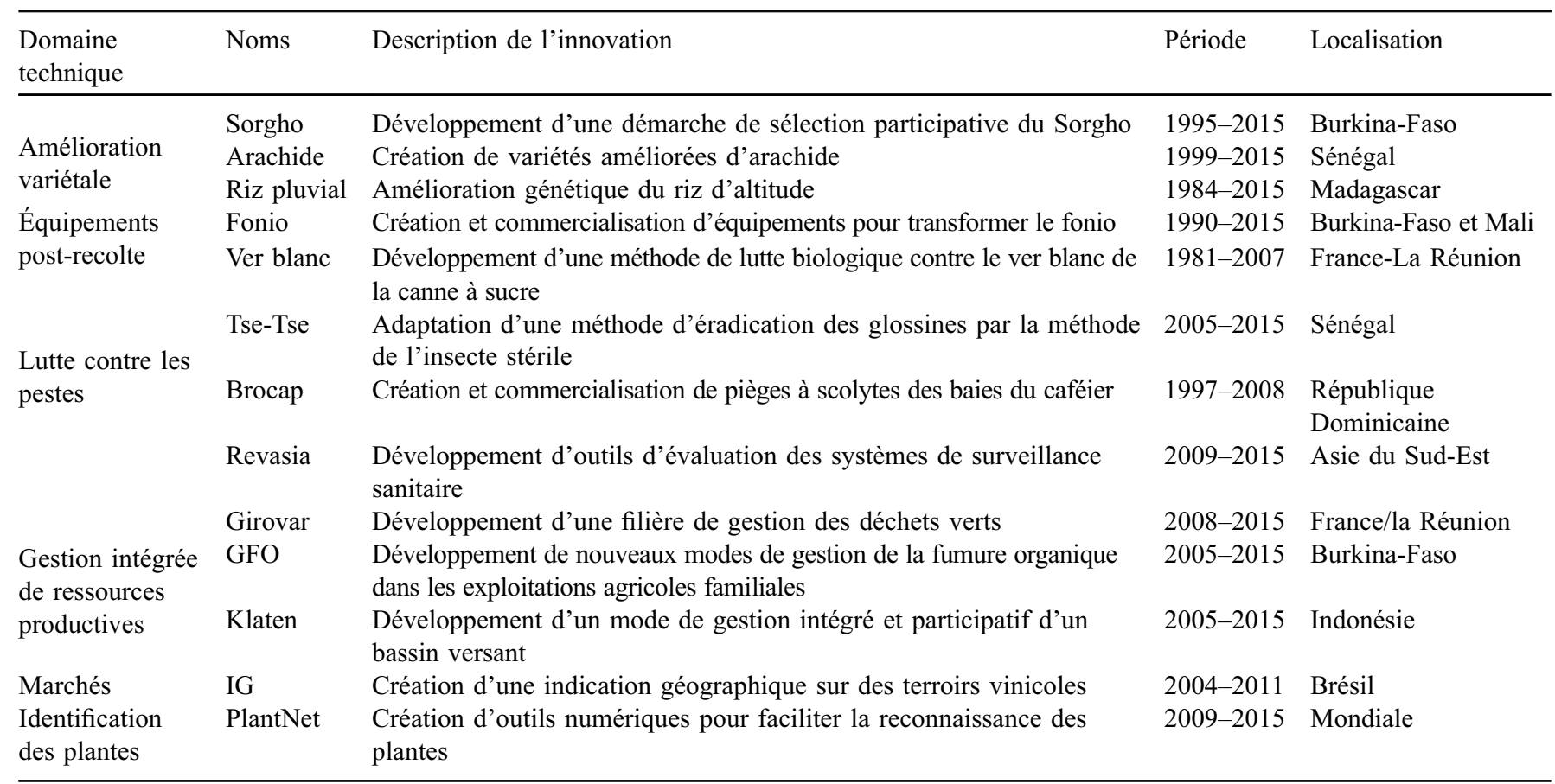

\section{Résultats}

\subsection{Diversité des situations d'apprentissage en lien avec chaque phase de l'innovation}

On montre dans un premier temps qu'il existe une relative diversité de situations d'apprentissage, classées selon leur finalité et l'étape du processus d'innovation (Tab. 4).

Les SA visant à explorer, évaluer et/ou programmer des activités sont principalement citées dans les phases d'initiation des processus d'innovation avec une posture très variable des chercheurs impliqués, avec une fréquence élevée comme formateur puis comme apprenant ou explorateur. Généralement, la formation est vue comme un moyen de proposer de nouvelles idées, de sensibiliser à l'intérêt d'une nouvelle approche, ce qui explique son usage pour initier des innovations. Dans la posture de l'apprenant ou de l'explorateur, le chercheur met en place des dispositifs de collecte de données pour mieux comprendre une situation problématique et identifier de nouvelles pistes de résolution du problème rencontré. Les SA liées à des comités de projet ou des cellules de coordination de projet qui sont mises en place pour 
Tableau 3. Sources des données primaires pour chaque étude de cas.

Table 3. Primary data sources for each case study.

\begin{tabular}{|c|c|c|c|c|}
\hline \multirow[t]{2}{*}{ Cas } & \multicolumn{3}{|c|}{ Source des données primaires } & \multirow{2}{*}{$\begin{array}{l}\text { Nombre de } \\
\text { types de SA } \\
\text { caractérisées }\end{array}$} \\
\hline & $\begin{array}{l}\text { Nombre ateliers } \\
\text { multi-acteurs } \\
\text { d'auto-évaluation }\end{array}$ & $\begin{array}{l}\text { Nombre entretiens } \\
\text { et focus groupes }\end{array}$ & Catégories d'acteurs interrogés & \\
\hline Sorgho & 3 & 41 entretiens & $\begin{array}{l}\text { Chercheurs } \\
\text { Agriculteurs, collectifs paysans locaux } \\
\text { Techniciens de recherche }\end{array}$ & 5 \\
\hline Riz pluvial & 2 & 232 entretiens & $\begin{array}{l}\text { Chercheurs } \\
\text { Agriculteurs } \\
\text { Techniciens; Agent de vulgarisation; Formateurs ; Consultants ; } \\
\text { Responsables d'instituts techniques et chefs de service } \\
\text { Distributeurs; Commerçants ; Décortiqueurs }\end{array}$ & 10 \\
\hline Ver blanc & 0 & $\begin{array}{l}69 \text { entretiens } \\
4 \text { focus group } \\
\text { (planteurs de } \\
\text { cannes à sucre; } \\
\text { services publiques) }\end{array}$ & $\begin{array}{l}\text { Chercheurs } \\
\text { Planteurs; Organisations agricoles (syndicats, organisation } \\
\text { de producteurs) } \\
\text { Services publics (directions, chambre d'agriculture, conseil } \\
\text { général, mairie) } \\
\text { Distributeurs }\end{array}$ & 8 \\
\hline Tse-Tse & 1 & $\begin{array}{l}63 \text { entretiens } \\
1 \text { focus group } \\
\text { (éleveurs, } \\
\text { vétérinaire, } \\
\text { chercheurs) }\end{array}$ & $\begin{array}{l}\text { Chercheurs } \\
\text { Éleveurs ; Responsable d'association d'éleveurs } \\
\text { Techniciens } \\
\text { Enseignants } \\
\text { Services du Ministère de l'Élevage et des Productions } \\
\text { animales } \\
\text { Acteurs du développement }\end{array}$ & 5 \\
\hline Revasia & 2 & $\begin{array}{l}38 \text { entretiens } \\
\text { Plusieurs focus } \\
\text { groups multi- } \\
\text { acteurs }\end{array}$ & $\begin{array}{l}\text { Chercheurs } \\
\text { Éleveurs } \\
\text { Services vétérinaires nationaux } \\
\text { Autorités locales } \\
\text { Institutions de surveillance sanitaire, organisations } \\
\text { internationales }\end{array}$ & 11 \\
\hline Girovar & 1 & 23 & $\begin{array}{l}\text { Chercheurs } \\
\text { Agriculteur; Éleveur } \\
\text { Entreprises de recyclage; Coopérative } \\
\text { Agents des Services publics (Conseil général) }\end{array}$ & 3 \\
\hline GFO & 2 & $\begin{array}{l}48 \text { entretiens } \\
7 \text { focus group } \\
\text { (producteurs) }\end{array}$ & $\begin{array}{l}\text { Chercheurs; Techniciens d'instituts de recherche } \\
\text { Formateurs et enseignants } \\
\text { Agriculteur } \\
\text { Cadres et élus d'organisations de producteurs } \\
\text { Techniciens d'agences de développement } \\
\text { Bailleur } \\
\text { Agents des services publics (collectivités locales, } \\
\text { ministères) }\end{array}$ & 6 \\
\hline
\end{tabular}


Tableau 3. (suite).

\begin{tabular}{|c|c|c|c|c|}
\hline Cas & \multicolumn{3}{|c|}{ Source des données primaires } & $\begin{array}{l}\text { Nombre de } \\
\text { types de SA } \\
\text { caractérisées }\end{array}$ \\
\hline Klaten & 2 & $\begin{array}{l}40 \\
4 \text { focus groups } \\
\text { (agriculteurs) }\end{array}$ & $\begin{array}{l}\text { Chercheurs } \\
\text { Agriculteurs } \\
\text { Associations d'usagers de l'eau et d'irrigants } \\
\text { Agents des services publics }\end{array}$ & 16 \\
\hline IG & 2 & $\begin{array}{l}50 \\
43 \text { réponses à } \\
\text { questionnaire par } \\
\text { email }\end{array}$ & $\begin{array}{l}\text { Chercheurs } \\
\text { Agriculteurs, viticulteurs } \\
\text { Coordinateurs de projet } \\
\text { Agents de services publics, techniciens }\end{array}$ & 3 \\
\hline PlantNet & 2 & $\begin{array}{l}37 \\
719 \text { réponses à } \\
\text { questionnaire par } \\
\text { email }\end{array}$ & $\begin{array}{l}\text { Chercheurs; Responsables d'instituts de recherche } \\
\text { Ingénieurs du projet Pl@ntnet } \\
\text { Enseignants } \\
\text { Agents de services publics (direction régionale, } \\
\text { conservatoire des espaces naturels) } \\
\text { Chef de service d'entreprises spécialisées; Responsables de } \\
\text { réseaux botaniques; Membres d'associations }\end{array}$ & 11 \\
\hline
\end{tabular}

développer l'innovation, jouent un rôle important dans le renforcement des capacités de gestion, de coordination, de collaboration des chercheurs partenaires du Cirad et des organisations professionnelles des filières.

Les SA visant à mettre en pratique des idées, des nouvelles techniques ou des prototypes sont fréquentes dans les phases de développement de l'innovation. Les SA les plus fréquentes sont des dispositifs d'expérimentation qui impliquent les utilisateurs finaux de l'innovation, et sont centrés sur le renforcement de leurs capacités techniques. La posture des chercheurs se distribue de façon quasi équivalente entre entrepreneur, explorateur et intervenant.

Les SA ayant pour finalité l'explicitation, la confrontation ou la mise en récit d'expériences ou de résultats apparaissent aux trois étapes des processus d'innovation étudiés. Il peut s'agir de confronter une nouvelle idée à un public large pour tester sa pertinence avant de lancer son développement, ou de formaliser des analyses de données de façon à clarifier l'utilité des résultats et faciliter le changement d'échelle. Les chercheurs jouent généralement un rôle de formateur ou d'expert en appui à un groupe d'apprenants ou face à un grand public. On constate que les postures endossées par les chercheurs dépendent principalement des compétences disponibles dans le collectif engagé dans l'innovation, les chercheurs se substituant à des communicateurs ou facilitateurs, principalement lors des évènements grands public, des ateliers bilans et des voyages d'étude. Lorsque ces compétences sont présentes sur place, le chercheur est absent de ces SA.

\subsection{Quatre types de contribution des chercheurs au renforcement de capacité}

L'analyse des différentes séquences de SA et des fréquences de posture des chercheurs nous a permis d'identifier quatre types de contribution au renforcement des capacités à innover (Tab. 5). Chaque type est illustré par un cas d'étude (Fig. 5).
Le premier type de contribution consiste en une facilitation des apprentissages de façon non supervisée. C'est-à-dire que les chercheurs interviennent essentiellement comme formateurs à la demande auprès des différents groupes d'acteurs impliqués dans le développement de l'innovation, sans suivre de façon continue les progrès réalisés et les besoins d'apprentissage. Les capacités renforcées sont principalement celles de producteurs et d'organisations de producteurs et concernent des capacités techniques individuelles et des capacités à expérimenter (études de cas Arachide, Sorgho, Riz pluvial). Des capacités collectives de collaboration sont renforcées indirectement via les cellules d'animation de projet.

Le second type consiste en une planification et un encadrement des apprentissages pour le transfert et l'adaptation soit d'une nouvelle technologie (Ver blanc, Fonio) soit de dispositifs sociotechniques novateurs (IG, Tsé-Tsé). Les apprentissages visent la création progressive de compétences locales pour pérenniser le dispositif (capacités de gestion, de coordination, de suivi-évaluation) et concernent principalement les partenaires de recherche au Sud. Les chercheurs du Cirad sont principalement dans une posture d'entrepreneurs et guident fortement le processus d'appui à l'innovation.

Le troisième type est un accompagnement des apprentissages qui repose sur des méthodes participatives. Il est initié par un autodiagnostic des problèmes rencontrés et des besoins d'appui. Les chercheurs se positionnent principalement comme des intervenants et des formateurs qui vont accompagner au fur et à mesure les besoins d'apprentissage exprimés par les acteurs de l'innovation. Dans ce cas, le dispositif même d'accompagnement est innovant dans de nombreux contextes, comme le dispositif de recherche-action en partenariat utilisé dans le cas de gestion des fumures organiques au Burkina-Faso ou la modélisation d'accompagnement pour le cas Klaten en Indonésie. Les apprenants sont alors de deux sortes: les acteurs concernés par le produit de l'innovation (agriculteurs, éleveurs, services techniques) et les acteurs qui apprennent non pas sur l'innovation mais sur le 
Tableau 4. Diversité des situations d'apprentissage (SA) et fréquence des postures des chercheurs. Table 4. Diversity of learning situations (SA) and frequency of researchers' postures.



\footnotetext{
* Nombre de cas où la posture pour ce type de SA est citée/nombre total de cas où ce type de SA a été identifié.
}

dispositif d'appui à l'innovation et les outils utilisés (concertation, recherche-action en partenariat [RAP], évaluation participative), principalement les chercheurs partenaires au Sud.

Dans les types 2 et 3 , les capacités renforcées concernent principalement des capacités individuelles à s'engager dans l'action collective et des capacités de leadership. Les nouvelles capacités collectives portent sur la collaboration ou l'échange avec des acteurs inhabituels, généralement des services techniques publics ou des acteurs politiques.

Le dernier type repose sur des apprentissages par exploration, qui concernent principalement les chercheurs eux-mêmes. Dans ce cas, la recherche est leader du processus d'innovation dans la mesure où c'est elle qui est à l'origine de la nouvelle idée ou du nouveau produit dont elle a fait reconnaître l'utilité ; elle cherche ensuite à améliorer, breveter et commercialiser son produit en se laissant guider par les besoins des utilisateurs. Les principales capacités développées sont la capacité à créer des réseaux de développeurs et d'utilisateurs alignés sur le même objectif, ce qui a facilité le changement d'échelle, ainsi que la capacité à mobiliser les ressources nécessaires à chaque étape de l'innovation.

\section{Discussion}

\subsection{L'adaptabilité des chercheurs comme capacité émergente}

L'approche par les situations d'apprentissage a permis d'explorer la diversité des rôles endossés par les chercheurs, donnant ainsi une image de la nature du travail fourni aux différentes étapes de l'innovation. Elle confirme que la production de connaissances est loin d'être leur unique rôle.

Des nouvelles postures ont par ailleurs été identifiées: communicateur, facilitateur ou organisateur des mises en relations de différentes catégories d'acteurs (types 3 et 4), ce que Howells (2006) décrit comme des fonctions émergentes des intermédiaires dans les systèmes d'innovation. 
A. Toillier et al. : Cah. Agric. 2018, 27, 15002

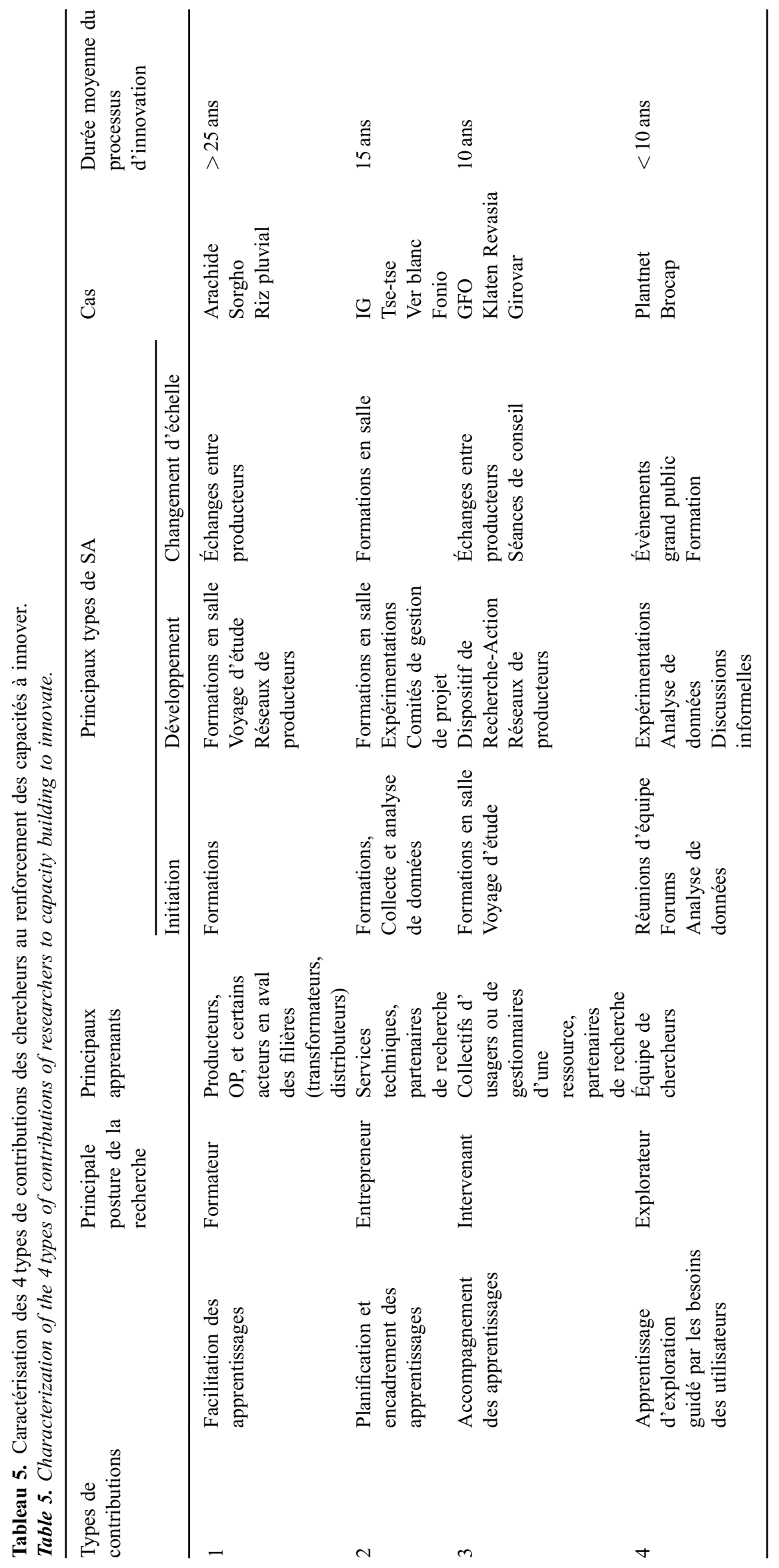

Page 9 de 13 
Type 1 : Cas « Sorgho ", principaux apprenants : producteurs, organisations de producteurs et distributeurs

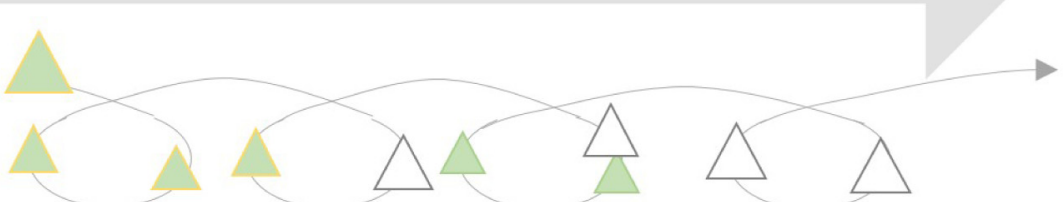

Type 2 : Cas « Tse-Tse », principaux apprenants : services vétérinaires et chercheurs

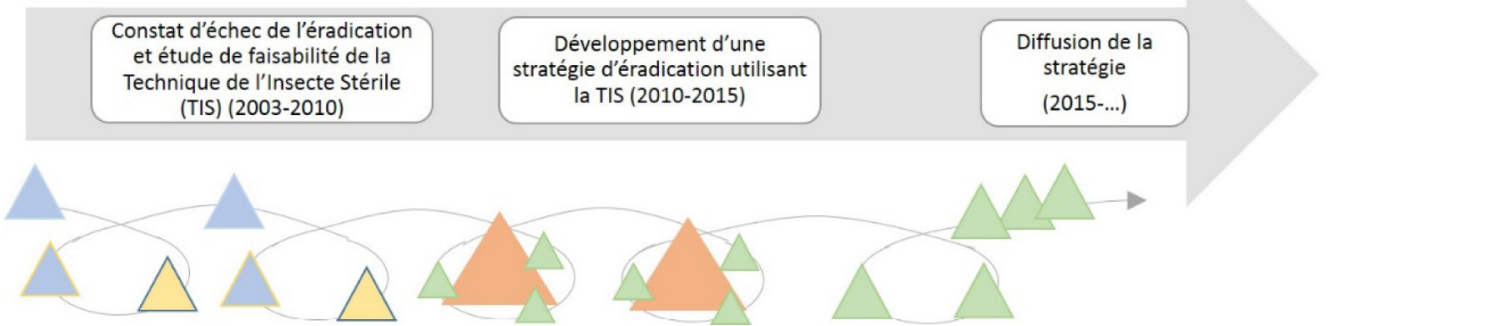

Type 3 : Cas " Gestion des fumures organiques ", Principaux apprenants : producteurs et chercheurs



\section{Type 4 : Cas « PlantNet », Principaux apprenants : chercheurs}

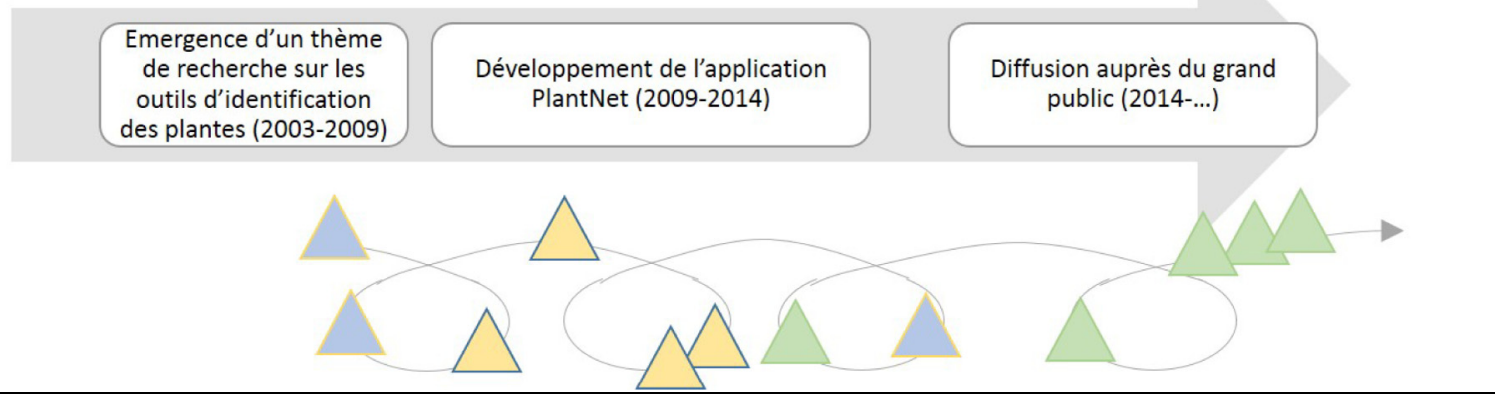

Posture dominante des chercheurs dans la situation d'apprentissage

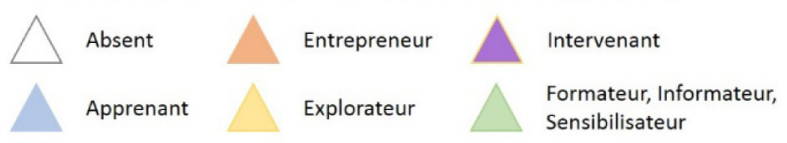

Fig. 5. Illustration des quatre types de contribution des chercheurs au RCI.

Fig. 5. Illustration of the four types of researchers' contribution to the RCI. 
Il apparaît les chercheurs peuvent endosser plusieurs postures au cours d'un processus d'innovation, grâce, par exemple, à une coordination entre le Cirad et les organismes de recherche partenaires qui facilite la multiplicité des rôles joués dans le type 3.

On note que la variabilité des postures des chercheurs n'est pas corrélée à un type d'innovation (technique, sociale organisationnelle ou de service), ni corrélée à l'étape du processus d'innovation, contrairement à ce que supposent Douthwaite et Gummert (2010). Il apparaît plutôt des changements de postures en fonction des enjeux de RCI que les chercheurs rencontrent dans un contexte donné pour atteindre les objectifs visés, selon les opportunités d'activités données par les projets de R\&D et les compétences disponibles sur place. Cette adaptabilité peut donc être très variable d'un contexte à l'autre, et conditionnée par les aptitudes même du chercheur à savoir s'adapter.

\subsection{Un large éventail de capacités pour de multiples acteurs sont nécessaires pour innover}

Les cas étudiés montrent que les capacités renforcées sont très diverses - technique (maîtriser une nouvelle technologie), managériale (suivre, évaluer, recruter, mobiliser des moyens), fonctionnelle (collaborer, expérimenter, mettre en réseau)concernent plusieurs niveaux (individu, organisation, collectif, projet) et concernent différents types d'acteurs selon l'innovation développée (producteurs, organisations de producteurs, chercheurs, techniciens des services publiques, artisans, etc.).

La littérature (Pant, 2012; Musiolik et al., 2012) montre que les capacités produites à chaque niveau contribuent à améliorer le fonctionnement des autres niveaux, ce qui explique le caractère itératif des situations d'apprentissage repérées par les acteurs ainsi que la mobilisation préférentielle d'individus lors des premières étapes de l'innovation, puis de réseaux et d'organisations aux phases de développement et de changement d'échelle. La méthode utilisée ne permet pas de rendre compte de la façon dont ce mécanisme (la façon dont le RCI à un niveau contribue aux RCI des autres niveaux) a lieu. En revanche il apparaît que dans les types de contribution 2 et 4 , ce sont les chercheurs qui coordonnent la production des capacités requises aux différents niveaux via les projets qu'ils pilotent; dans les types 1 et 3 , ils repèrent des besoins en renforcement de capacités, mais ne sont pas en position de coordination ou de gestion.

On distingue ainsi chez les chercheurs des apprentissages d'ordres différents, portant soit sur l'innovation même (comment améliorer une semence ou transformer le fonio), soit sur le processus d'appui à l'innovation en lui-même (comment organiser les acteurs en situation d'innovation et les échanges collaboratifs). Dans le premier cas (types 1 et 4), ce sont principalement des chercheurs du Cirad qui sont en posture d'apprenants alors que dans le second cas (types 2 et 3 ), ce sont plutôt les partenaires de recherche au Sud, formés par des chercheurs du Cirad. Cette observation interroge les spécificités d'une recherche, celle menée au Cirad, en appui à une autre recherche, celle des partenaires du Sud, dont on perçoit des fonctions particulières liées à un double positionnement: auprès des acteurs en train d'innover mais aussi auprès des acteurs en charge de l'appui à l'innovation, notamment les chercheurs.

\section{Conclusion: perspectives pour renforcer l'efficacité d'une recherche agricole en appui à l'innovation au Sud}

Les cas analysés fournissent deux résultats clés sur les différentes modalités de contribution des chercheurs à des processus d'innovation collective variés. D'une part, il existe une adaptabilité et flexibilité des chercheurs aux situations qu'ils rencontrent, qui se traduit par l'endossement de multiples postures qui leur permettent de contribuer au renforcement des capacités à innover dans des contextes très variés. D'autre part, la multiplicité des situations d'apprentissage nécessaires au renforcement de capacités individuelles et collectives menant à l'innovation, confirme l'importance du rôle de coordination et de mobilisation de partenaires de recherche et de développement que jouent les chercheurs du Cirad dans certains cas pour organiser l'appui à l'innovation.

Nos résultats ouvrent des perspectives pour renforcer l'efficacité d'une recherche agricole en appui à l'innovation au Sud.

Telle que conçue dans le programme ImpresS du CIRAD, l'évaluation des impacts de la recherche sur l'innovation et le développement visait essentiellement à susciter des changements chez les chercheurs en créant une « culture de l'impact», c'est-à-dire en les incitant à penser davantage, en amont de leurs activités, la façon dont ils peuvent contribuer efficacement à l'innovation. Nos résultats confirment qu'il y a nécessité pour les chercheurs de savoir s'adapter et être flexible pour adopter une posture adéquate selon le type de situation dans laquelle ils se trouvent et ainsi contribuer aux apprentissages et à l'innovation pour avoir de l'impact.

Pour cela, ces derniers doivent être incités à questionner eux-mêmes et faire évoluer leurs pratiques de R\&D. Pour les aider, il est nécessaire de mieux comprendre les circonstances qui les amènent à opter pour une posture ou une autre. Leurs choix sont-ils intentionnels, contraints par les projets de R\&D, guidés par leur savoir-faire ou par les engagements pris, ou encore imposés par leur hiérarchie? De quelles marges de manœuvre disposent-ils pour décider de leur positionnement dans une situation d'apprentissage ? Existe-t-il par exemple une corrélation avec leur discipline, leur sujet de recherche ou tout simplement avec le système de suivi-évaluation de leur institut qui s'impose à eux? Par exemple dans les cas où les chercheurs remplissent de multiples rôles, il peut s'agir d'une forme de prise de pouvoir intéressée puisque dans ces projets les chercheurs ne sont pas nécessairement qu'au service du processus d'innovation contrairement à la majorité des autres acteurs. Ils peuvent avoir des motivations propres liées à leur mandat (publication, responsabilité, honoraires). Dans d'autres cas, leur posture peut relever d'une volonté de pallier l'absence de compétences disponibles ou mobilisables pour remplir les engagements pris. En effet, la grande majorité des projets de $\mathrm{R} \& \mathrm{D}$ 《traditionnels » sont très souvent focalisés dans leur planification sur les enjeux techniques de l'innovation, laissant les besoins en renforcement de capacités fonctionnelles (collaborer, s'engager, évaluer, gérer, etc.) comme une variable d'ajustement laissée à l'appréciation des porteurs de projet une fois les financements acquis.

De nos résultats, des enseignements peuvent être tirés davantage pour les gestionnaires ou bailleurs des projets 
d'innovation que pour les chercheurs eux-mêmes. Ils mettent en évidence la multitude d'activités et de «(re)configurations partenariales » dans chaque SA (tous les acteurs ne sont pas mobilisés de la même manière ni au même moment) qui doivent avoir lieu pour que des capacités soient renforcées et que l'innovation réussisse. La durée moyenne observée pour chacun des types de mécanismes de RCI interroge l'efficacité de certaines formes de contribution des chercheurs et laisse penser qu'une gestion stratégique pourrait permettre de réduire la durée de certaines étapes (Cohendet et al., 2008). Repérer le mécanisme à l'œuvre pourrait permettre de rationaliser le temps des chercheurs en mobilisant d'autres métiers nécessaires pour appuyer l'innovation, tels que des facilitateurs de l'innovation (Klerkx et al., 2009) ou des designers de l'innovation (Szostak, 2006). Cette perspective incite à repenser plus globalement la nature des compétences et des mandats des chercheurs engagés dans la gestion ou la coordination de projets d'innovation : doit-il y avoir, au sein des organismes de recherche, des chercheursaccompagnateurs de l'innovation, capables de produire des connaissances gestionnaires sur un processus d'innovation en cours et d'aider l'optimisation du dispositif de pilotage de l'innovation chemin-faisant?

Pour les organismes de recherche, les résultats de cette étude pourraient être utilisés comme support de formation des chercheurs qui ont été impliqués dans les différents cas d'innovation afin de renforcer leur culture de l'impact. Il s'agirait de développer chez les individus, capacités de réflexivité et pensée complexe, qui, d'après Senge (2016), facilitent l'innovation collective. Si chacun a une vision globale des mécanismes qui fondent un processus d'innovation collective, cela permet à chaque individu d'ajuster ses contributions de façon à nourrir la progression collective et ainsi contribuer à l'innovation. Une autre possibilité serait d'organiser plus systématiquement des évaluations participatives ex post de projets d'innovation. Elles sont reconnues comme ayant des vertus endo-formatrices (Plottu et Plottu, 2009) et peuvent être utilisées à dessein pour créer des changements chez les participants dans leur manière de contribuer ultérieurement, dans d'autres circonstances, aux résultats initialement visés et qui font l'objet de l'évaluation.

\section{Références}

Audoux C, Gillet A. 2011. Recherche partenariale et co-construction de savoirs entre chercheurs et acteurs : l'épreuve de la traduction. Revue Interventions économiques. Papers in Political Economy 43. Disponible sur http://interventionseconomiques.revues.org/1347.

Beckman SL, Michael B. 2007. Innovation as a Learning Process: Embedding Design Thinking. California Management Review 10 50(1): 25-56.

Cohendet P, Grandadam D, Simon L. 2008. Réseaux, communautés et projets dans les processus créatifs. Management International 13(1): 29-43.

Delma BJ, Nacro HB, Bougouma-Yameogo V, Vall E. 2016. Conception participative d'innovations et accompagnement des projets d'élevage familiaux au Burkina-Faso: explorer, expérimenter et évaluer. Revue d'Élevage et de Médecine Vétérinaire des Pays Tropicaux 69(2): 63-71.

Dimaggio PJ. 1988. Interest and Agency in Institutional Theory. In : Zucker LG, dir. Institutional Patterns and Organizations: Culture and Environment. Cambridge (Ma): Ballinger Editions, pp. 3-22.
Douthwaite B, Gummert M. 2010. Learning selection revisited: How can agricultural researchers make a difference? Agricultural Systems 103: 245-255.

Draetta L, Labarthe F. 2011. La recherche sur commande et le dilemme expert-chercheur: une analyse réflexive de l'implication $\mathrm{du}$ sociologue dans la mise en œuvre d'une politique publique de l'innovation. Revue Interventions économiques 43. Disponible sur http://interventionseconomiques.revues.org/1425.

Engestrom Y. 1999. Innovative learning in work teams: Analyzing cycles of knowledge creation in practice. Perspectives on Activity Theory: 377-404.

Faure G, Gasselin P, Triomphe B, Temple L, Hocdé H. 2010. Innover avec les acteurs du monde rural : la recherche-action en partenariat. Versailles : Éditions Quae, 224 p.

Foray D. 1994. Recherche et technologie - Les nouveaux paradigmes de l'apprentissage technologique. Revue d'Économie Industrielle 69( $3^{\mathrm{e}}$ trimestre): 93-104.

Hainzelin E, Barret D, Faure G, Dabat M-H, Triomphe B. 2017. Agricultural research in the Global South: steering research beyond impact promises. Montpellier: CIRAD, Perspective 42, 4 p.

Hall A. 2005. Capacity development for agricultural biotechnology in developing countries: An innovation systems view of what it is and how to develop it. Journal of International Development 17: 611630 .

Hall R, Andriani P. 2003. Managing knowledge associated with innovation. Journal of Business Research 56: 145-152.

Hatchuel A. 1994. Apprentissages collectifs et activités de conception. Revue Française de Gestion 99: 10-120.

Howells J. 2006. Intermediation and the role of intermediaries in innovation. Research Policy 35(5): 715-728.

Klerkx L, Hall A, Leeuwis C, 2009. Strengthening agricultural innovation capacity: are innovation brokers the answer? International Journal of Agricultural Resources, Governance and Ecology 8(5-6): 409-438.

Klerkx L, van Mierlo B, Leeuwis C. 2012. Evolution of systems approaches to agricultural innovation: concepts, analysis and interventions. In: Darnhofer I, Gibbon D, Dedieu B, eds. Farming Systems Research into the 21st Century: The New Dynamic. Springer Science (Dordrecht), pp. 457-483.

Kolb DA, Boyatzis RE, Mainemelis C. 2001. Experiential learning theory: Previous research and new directions. Perspectives on Thinking, Learning, and Cognitive Styles 1(8): 227-247.

Lamers D, Schut M, Klerkx L. 2017. Compositional dynamics of multilevel innovation platforms in agricultural research for development. Science and Public Policy: 1-14.

Laperche B, Munier F, Hamdouch A. 2008. The collective innovation process and the need for dynamic coordination: general presentation. Journal of Innovation Economics \& Management 2(2): 3-13.

Leeuwis C, Schut M, Waters-Bayer A, Mur R, Atta-Krah K, Douthwaite B. 2014. Capacity to innovate from a system CGIAR research program perspective. Program Brief: AAS-2014-29. CGIAR Research Program on Aquatic Agricultural Systems, Penang, Malaysia.

Lusthaus C, Adrien M-H, Perstinger M. 1999. Capacity development: definitions, issues and implications for planning, monitoring and evaluation. Universalia Occasional paper $n^{\circ} 35$, septembre 1999, 21 p.

Martin A, 2013. Le Partenariat Européen pour l'Innovation (PEI) : la mise en réseaux comme levier de l'innovation en agriculture. Centre d'Études et de Prospective, $\mathrm{n}^{\circ}$ 65, novembre 2013, 4 p.

Musiolik J, Markard J, Hekkert M. 2012. Networks and network resources in technological innovation systems: Towards a conceptual framework for system building. Technological Forecasting and Social Change 79: 1032-1048. 
Pant LP. 2012. Learning and Innovation Competence in Agricultural and Rural Development. The Journal of Agricultural Education and Extension 18: 205-230.

Perez YA. 2008. La pratique de la recherche-intervention dans les organisations: retour sur les modes de production des connaissances gestionnaires à partir du terrain. Humanisme et Entreprise 3 (288): 101-113.

Petticrew M, Roberts H. 2003. Evidence, hierarchies and typologies: horses for courses. Journal of Epidemiology and Community Health 57: 527-9.

Pichault F, Lisein O, Rondeaux G, Xhauflair V. 2008. La Rechercheintervention peut-elle être socialement responsable? Paris: Éditions Vuibert, $251 \mathrm{p}$.

Plottu B, Plottu E. 2009. Contraintes et vertus de l'évaluation participative. Revue française de gestion 2(192): 31-58.

Sabourin E, Da Silveira LM, Sidersky P. 2004. Production d'innovation en partenariat et agriculteurs-expérimentateurs au Nordeste du Brésil. Cahiers Agricultures 13(2): 203-210.

Senge P. 2016. La cinquième discipline. Levier des organisations apprenantes. Eds Eyrolle, 460 p.

Spielman DJ, Ekboir J, Davis K, 2009. The art and science of innovation systems inquiry: Applications to Sub-Saharan African agriculture. Technology in Society 31: 399-405.

Szostak B, 2006. La profession de designer. Une source légitime de créativité. Revue française de gestion 2(161): 125-138.

TAP (Tropical Agriculture Platform). 2016. Common Framework on Capacity Development for Agricultural Innovation Systems: Conceptual Background. Wallingford (UK): CAB International, $101 \mathrm{p}$.
Toillier A, De Lapeyre de Bellaire L, 2016. Contributions de la recherche à l'innovation dans les filières. In : Biénabe E, Rival A, Loeillet D, eds. Développement durable et filières tropicales. Versailles: Ed. Quae, pp. 97-108.

Triomphe B, Barret D, Clavel D, Dabat MH, Devaux-Spatarakis A, Faure G, et al. 2015. Towards a generic, comprehensive and participatory approach for assessing the impact of agricultural research in developing countries. In: Impacts of Agricultural Research - an Approach of Societal Values. Paris: INRA, 27 p. Available from https://colloque.inra.fr/impar.

Turner JA, Klerkx L, Rijswijk K, Williams T, Barnard T, 2016. Systemic problems affecting co-innovation in the New Zealand Agricultural Innovation System: Identification of blocking mechanisms and underlying institutional logics. NJAS - Wageningen Journal of Life Sciences 76: 99-112.

Vall E, Chia E, Blanchard M, Koutou M, Coulibaly K, Andrieu N. 2016. La co-conception en partenariat de systèmes agricoles innovants. Cahiers Agricultures 25: 15001.

Woodhill J. 2010. Capacities for institutional innovation: a complexity perspective in capacity, reflecting collectively on capacities for changes. IDS Bulletin 41(3): 47-59.

World Bank. 2007. Enhancing agricultural innovation: how to go beyond the strengthening of research systems. Washington DC, $135 \mathrm{p}$.

Xhauflair V, Pichault F. 2011. Le chercheur comme entrepreneur de l'innovation inter-organisationnelle. Enjeux méthodologiques d'une recherche-intervention partenariale. SociologieS. Dossiers, Les partenariats de recherche.

Citation de l'article : Toillier A, Devaux-Spartakis A, Faure G, Barret D, Marquié C. 2018. Comprendre la contribution de la recherche à l'innovation collective par l'exploration de mécanismes de renforcement de capacité. Cah. Agric. 27 : 15002. 